\title{
Smart Way to Track the Location in Android Operating System
}

\author{
Muthumurugesan $\mathrm{D}^{1}$, Nalini $\mathrm{S}^{2}$, Vinodini $\mathrm{R}^{3}$ \\ ${ }^{1,3}$ PG student, University College of Engineering, BIT Campus, Anna University, Thiruchirappalli \\ ${ }^{2}$ Assistant Professor, University College of Engineering, BIT Campus, Anna University, Thiruchirappalli
}

\begin{abstract}
A Location Based Service provides information that is accessible in the mobile devices through the mobile network and it also provides information about the contemporary geographical position of the mobile device. The user need not want to carry the desktop all the way along the journey to retrieve the details of destination location, to overcome this issue this application is used to extort the information about the mobile device which is integrated with Google Maps, Network provider and GPS to retrieve the latitude and longitude value. LBS use GPS to locate user's current location and user can select the new destination of their interest or they can choose the predetermined destination location which is closer to the current location. Through Query processing the user can retrieve the current location. The smart location tracker is deployed in the Android Operating system.
\end{abstract}

Keywords- LBS, GPS, Distance Calculator, Google Maps, Android

\section{Introduction}

A location Based Service (LBS) is a software application for an IP-capable mobile device that requires knowledge about where the mobile device is currently located. the system never allowed the user to access the mobile hardware directly. Now the user can access the mobile device hardware directly after the release of android based open source mobile phone. The basic components of LBS are service provider's software application, a mobile network to transmit data and requests for service, a content provider to supply the end user with geo-specific information, a positioning component (GPS), and the end user's mobile device.Location Based Service provides the users information services which originate from the geographic location of the end user mobile device. Using these services it is possible for the users to find current location and locate other persons, vehicle, resources .It also used to provide location-sensitive services, in addition to tracking their own location. The request for location can originate in the mobile device or network operator. It is feasible to automatically trigger current Location when the mobile device is at a particular location. Moreover additionally when a GPS is not available, query-based operation provides the end user with useful information such as current Location.Mobile devices are commonly equipped with GPS receivers. Because of the many satellites orbiting the earth, user can use a GPS receiver to find current location easily. However, GPS requires a clear sky to work and hence does not always work indoors otherwise where satellites can't penetrate .Another effective way to locate user position is through mobile tower triangulation. When a mobile is switched on, it is constantly in contact with base stations and surrounding it. By knowing the identity of cell towers, it is possible to translate this information into a physical location through the use of various databases containing the mobile signals' identities and their correct geographical locations. The advantage of mobile signal triangulation is that it works indoors, without the need to obtain information from satellites. However, it is not as worth as GPS because its accuracy depends on overlapping signal coverage, which varies quite a bit. Cell phone tower triangulation works best in densely populated areas where the cell towers are closely located.

\section{Location Services}

\subsection{Global Positioning System}

The Global Positioning System (GPS) is a space-based satellite navigation system that provides location and time information in all weather conditions, anywhere on or near the Earth where there is an unobstructed line of sight to four or more GPS satellites. The GPS (Global Positioning System) technology has been brought to the cell phones.

\subsubsection{Working of a GPS System}

GPS uses 27 satellites (24 currently working, 3 for backup) to enable a user to pin-point his or her current location. The calculation to ascertain the location is based on a arithmetic theory known as trilateration. Since the Earth is a sphere, each satellite generates a specific part of the sphere it hovers and revolves with. An intersection of three such spheres which is closest to the GPS device's locations done and the location is thus identifiedGPS gather the requesting device's current location and provide accurate response; the GPS receiver requires two vital details, i.e. the location of at least three satellites. Many mobile phones like an android mobile 
and tablets have integrated GPS (Global Positioning System) tracking systems that provide independent mobile tracking through the device alleviating the need for a separate dedicatedGPS unit.

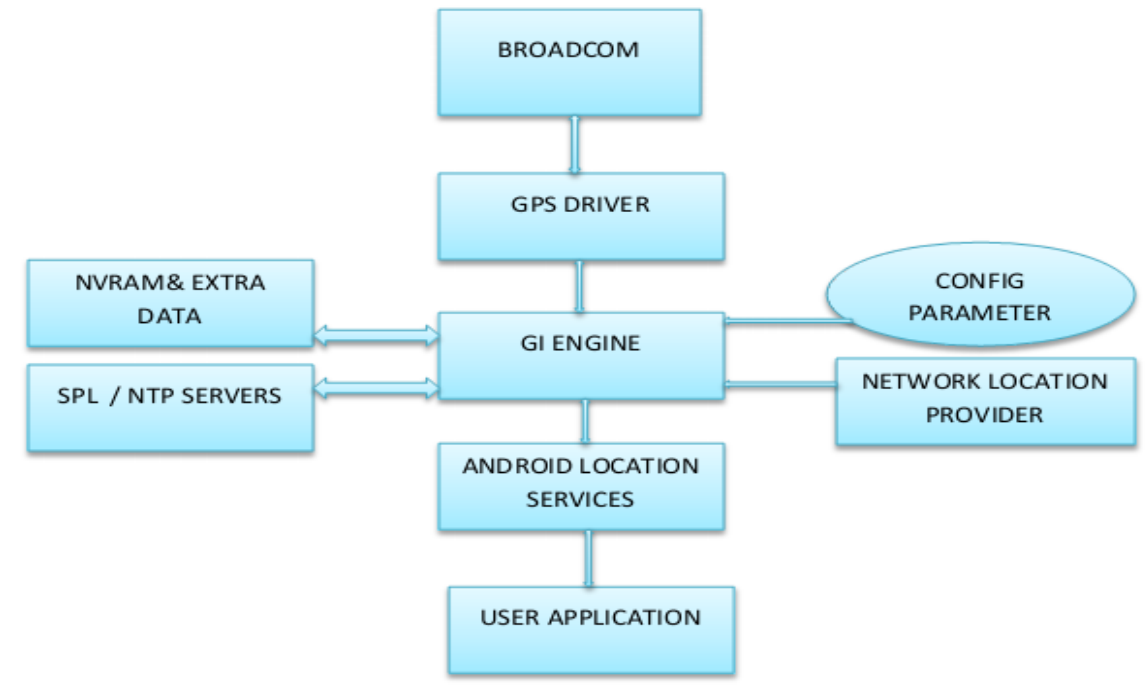

Fig 1.GPS working

GPS tracking in mobile phones was used to track users' locations. GPS tracking services are often referred to as location-based services. GPS provider and Network provider are most common location providers .They can be accessed by using the static string constants LocationManager.GPS_PROVIDER and LocationManager.NETWORK_PROVIDER

To access a specific provider getProvider() method can be used

String provider $=$ LocationManager.GPS_PROVIDER;

LocationProvider gps;

gps = locationManager.getProvider(provider);

GPS device its location will also be updated as soon as user changes his/her position.

\section{Distance Calculator}

The distance calculator is calculated by using the longitude and latitude values. The current location and destination place is has the two parameters value to find the distance between the places. The current location takes as the starting values and the destinations places is takes as the ending values. Using, the longitude and latitude the distance is calculated for the pre-defined places by distanceTo() method. The user can also enter the destination value and find the distance between the places. It will automatically show the kilometers between both the places.

Now the user has two array having latitude and longitude of nearby places and also has the user location latiude and longiude now the user can calculate the distance between user location and nearby places . In Android OS the mobile user can calculate the distanca through the stanceTo() method or distanceBetween method.Example for distanceTo() method is,

Location $\mathrm{x}=$ new Location("val1");

x.setLatitude(latx);

x.setLongitude(lngx);

Location $\mathrm{y}=$ new Location("val2");

y.setLatitude(laty);

y.setLongitude(lngy);

float distance $=\mathrm{x}$.distanceTo $(\mathrm{y})$;

Using SQLite database user can calculate the list of destination places and then it using math() function and round function. It can be coverted to kilometer using divided 1000.

$\mathrm{db}=$ openOrCreateDatabase("muthu", MODE_PRIVATE, null);

for (int i $=0$; i $<$ Place.length; i++)

\{locationB.setLatitude(latitude[i]);

locationB.setLongitude(longitude[i]);

distance $[\mathrm{i}]=$ Math

.round(locationA.distanceTo(locationB) / 1000);

\} 


\section{Google Maps In Android}

Google Maps is a mapping application provided by Google that can be embedded into mobile Siteby using the Google Map asset and Google Map Application Programming Interface (API). A Google Map can be used, for example, as a navigational tool for displaying your business locations or for allowing users to identify where they are visiting our site .Google Maps is a Web-based service that provides detailed information about geographical regions and sites around the world. In addition to conventional road maps, Google Maps offers aerial and satellite views of many places. In some cities, Google Maps offers street views comprising photographs taken from vehicles. It consists two major Component they are Google Map and Google Map Location

\subsection{Steps to generate API Key V2}

Generate keytool by using API download.

Open keytool and select create new key and create SHA-1 fingerprint values.

Using SHA_1 values signup in console to generate the API key.

In console create new key by using SHA-1 values but the SHA- 1 values must have package name at the end of value separated by colon.

Example,

34:F7:2C:0B:02:DF:E0:8F:0C:2A:99:9B:89:65:FE:16:C0:42:2B:EB;com.map.google

Then it will generate API key as,

AIzaSyBdVl-cTICSwYKrZ95SuvNw7dbMuDt1KG0

After key generation user has to get permission in Manifestfile as,

<uses-permissionandroid:name="android.permission.INTERNET"/>

<uses-permissionandroid:name="android.permission.ACCESS_NETWORK_STATE"/>

<uses-permissionandroid:name="android.permission.WRITE_EXTERNAL_STORAGE"/>

<uses-permissionandroid:name="com.google.android.providers.gsf.permission.READ_GSERVICES"/>

Version2 of the Google Maps Android API requires OpenGL ES version 2, user must add a <uses feature> element as a child of the <manifest> element in AndroidManifest.xml:

<uses-feature android:glEsVersion="0x00020000"android:required="true"/>

Now run the Android Apps, the Google maps is generated.

\section{Design Of Location Tracking}

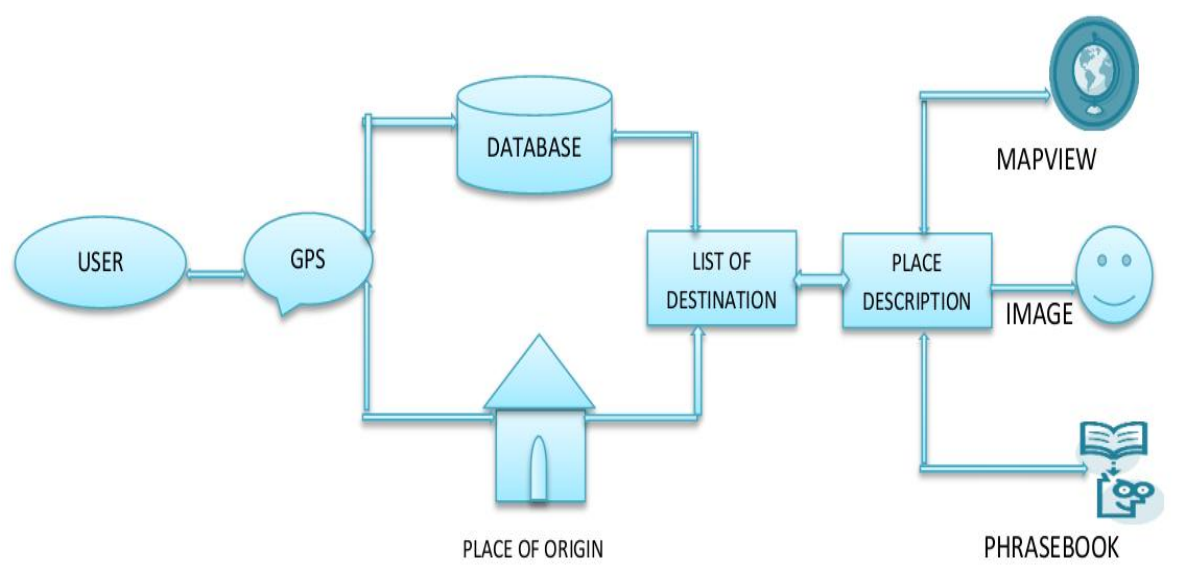

Fig 2.Design Of Smart Location Tracker

In this proposed work, the application is integrated with the GPS network providers, Google maps and some of the information is provide through the database and it will indicate the place of the origin. Location based service finds the current location of the user and it will list out the destinations and the place these process list the destination places by calculating the distance from the current place when GPS is available. Each place contains the image, name, rating, theme and distance from current location automatically. By default the list is sorted by distance. The list can also be sorted based on rating, alphabetical, theme and distance.

Now, the users can filter the desired destination. Two options are provided to filter the destinations. One is State wise and another one is Theme wise. Users can select multiple states and themes. It can be also sorted by ratings, themes, and distance and alphabetical. Otherwise, the user gives the destination place and 
it will automatically calculate the distance between the current place and destination the place. This process contains the list of predefined places where user can select their starting location by typing the place name and can find the destinations distance from that place. They can select their location from the list when the GPS is not available. Additionally, the user can use the map view, phrase book and picture view to get the details about the destination place.Each and every destination has a Detail view and Most Visited places details. Applications also contains a gallery view for each destinations, Phrase book for Language Helper and Map View for that destination places with geographical view

\section{Implementation And Result}

The current location will be fetched when the Apps Starts using GPS and it takes few seconds to fetch which is represented in the (fig.3).

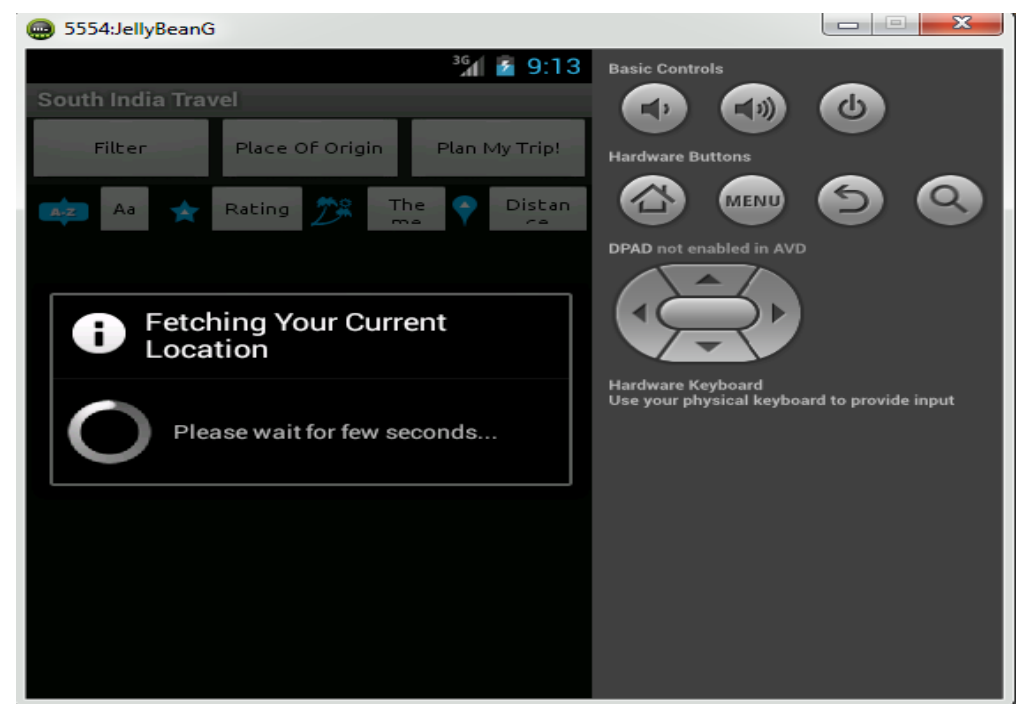

Fig 3.Fetching Current Location

The list of destinations will be listed in listview.In listview the current location takes as zero $(0) \mathrm{km}$ and then destination places distance will be generated automatically from the current location using latitude and longitude values.The destinations are shown(fig.4) with their coressponding distance. User can sort the destinations by Alphabetical, Ratings, Themes and Distance wise .

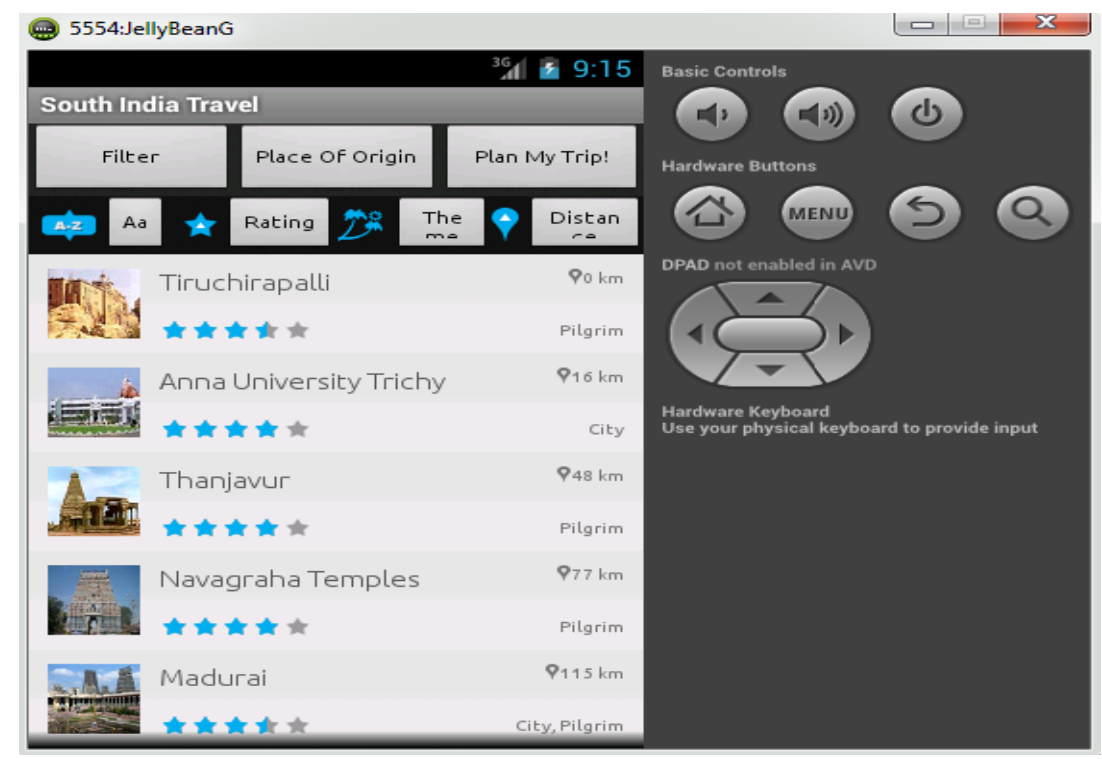

Fig 4. Destination List

User can filter the destinations based on Statewise or Themes such Hillstation ,Beaches and etc. the end user can select multiple options in the themesThen the result display with their distance which was selected by user. 


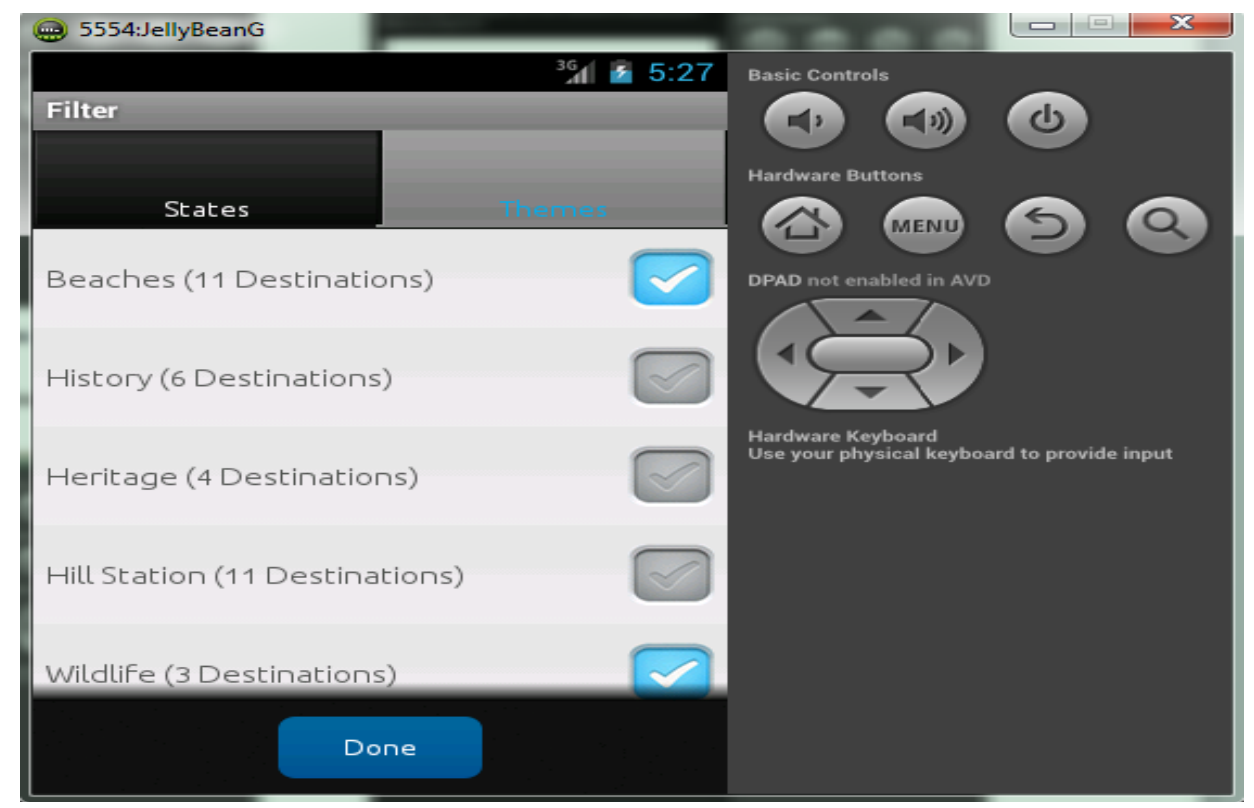

Fig 5. Filter

User can use a Query processing to find out the destinations when GPS is not available.Here the user can't access a current location because missing of GPS. Its show below(see fig.6).

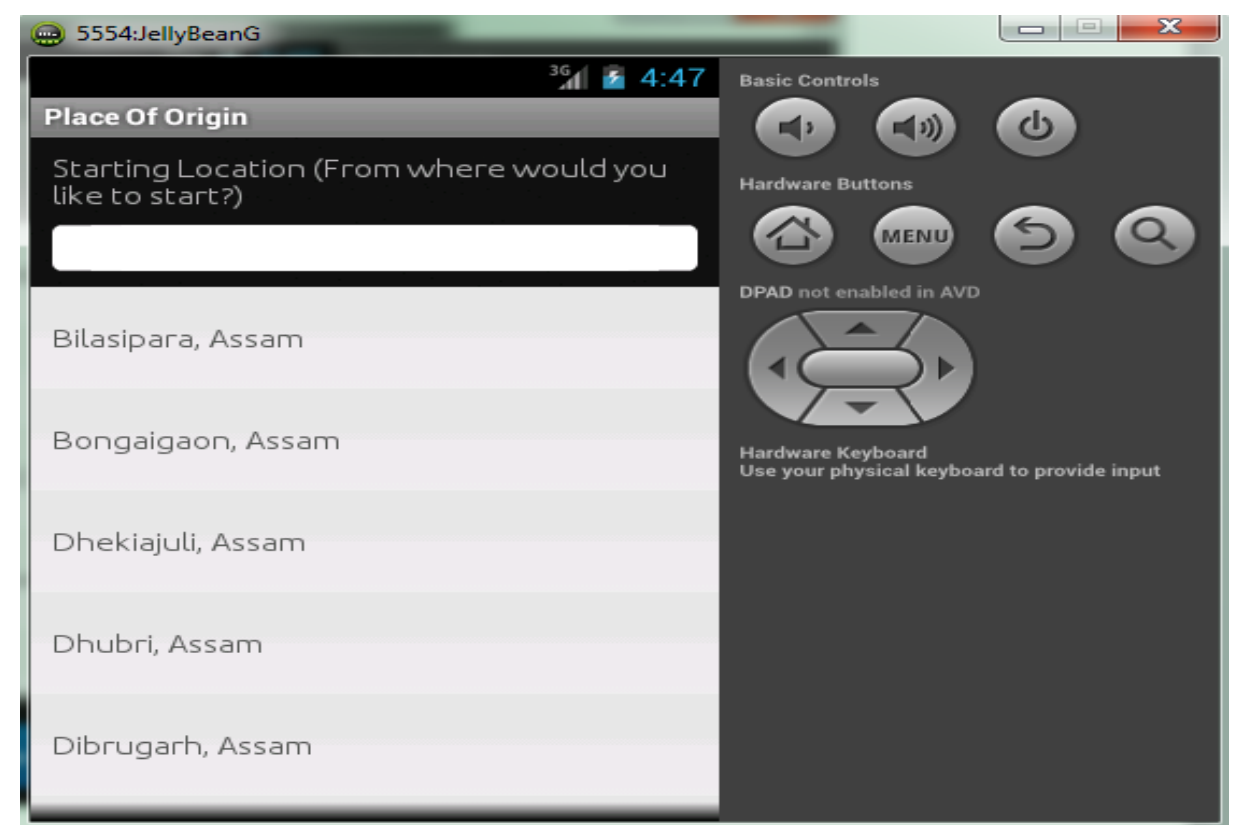

Fig 6.Query Processing

\section{Conclusion}

Location Based Services are those services which provide information and are accessible via mobile devices through the mobile network. They utilize the ability to make use of the geographical position of the mobile device. LBS can utilize some technologies such as the GPS, WiFi, Cellular networks. These services fetch the current location using GPS and in the absence of GPS using query processing the end user can identify the destination place. This application also provides destination distance from the current location, additionally it contains the Phrase book to language interpretation, and Map view to all destinations with Most visited places with details view about destinations. This application is a real time travel helper for the end user based on Android mobile devices. 


\section{References}

[1] Ch. Radhika Rani and A. Praveen Kumar,"LOCATION BASED SERVICES IN ANDROID", International Journal of Advances in Engineering \& Technology, March 2012.

[2] Gurjeet Kaur and Monika Sachdeva,"Mobile Client's Access Mechanism for Location based Service using Cell-ID ",International Journal of Computer Applications (0975 - 8887) Volume 57- No.22, November 2012.

[3] J.F. DiMarzio “Android TMA Programmer's Guide”.

[4] Shane Conder and Lauren Darcey "Android ${ }^{\mathrm{TM}}$ Wireless Application development", Second Edition.

[5] Katina Michael, Andrew McNamee and MG Michael," The Emerging Ethics of Human centric GPS Tracking and Monitoring", School of Information Technology and Computer Science and University of Wollongong, Australia.

[6] Gurjeet Kaur, Monika Sachdeva and Navdeep Singh" Mobile Client's Access Mechanism for Location based Service using Cell-ID “ in International Journal of Computer Applications (0975 - 8887) Volume 57- No.22, November 2012

[7] Manav Singhal1 and Anupam Shukla " Implementation of Location based Services in Android using GPS and Web Services" in IJCSI International Journal of Computer Science Issues, Vol. 9, Issue 1, No 2, January 2012 ISSN.

[8] Ch. Radhika Rani1, A. Praveen Kumar2, D. Adarsh2, K. Krishna Mohan2, K.V.Kiran2 "LOCATION BASED SERVICES IN ANDROID" International Journal of Advances in Engineering \& Technology, March 2012.

[9] Jason Ostrander “Android UI Fundamentals Develop and Design".

[10] RetoMeier "Professional Android" Application Development". 